\title{
Short hairpin RNA- mediated gene knockdown of FOXM1 inhibits the proliferation and metastasis of human colon cancer cells through reversal of epithelial-to-mesenchymal transformation
}

\author{
KanKan Yang ${ }^{1+}$, LinHua Jiang ${ }^{1 \dagger}$, You Hu${ }^{2}$, Jing Yu', HenFeng Chen ${ }^{1}$, YiZhou Yao ${ }^{1}$ and XinGuo Zhu ${ }^{1 *}$
}

\begin{abstract}
Background: The Forkhead box M1 (FOXM1) is an oncogenic transcription factor and plays a significant role in cell EMT, proliferation, metastasis in a multitude of human solid tumors including colorectal cancer (CRC). However, the underlying molecular mechanisms by which FoxM1 contributes to epithelial-to-mesenchymal (EMT) and metastasis have not been fully elucidated in CRC.

Methods: In our study, we investigated FOXM1 protein expression in 87 CRC tissue specimens, invasive lymph nodes and adjacent paired normal colorectal tissues by immunohistochemical analysis. Then we transfected FOXM1 specific shRNA into SW620 cells to examine effect of FOXM1 on proliferation, colony formation, migration and invasion in vitro. Western blotting and real-time PCR were used to detect the protein and mRNA expression of FOXM1 and EMT-related markers.
\end{abstract}

Results: FOXM1 was overexpressed in CRC tissues, invasive lymph nodes and CRC cell lines. FoxM1 overexpression was significantly associated with lymph node metastasis $(P<0.001)$, and tumor recurrence $(P<0.001)$. Moreover, downregulation of FOXM1 in SW620 cells by shRNA approach inhibited cell growth, clonogenicity, migration and invasion in vitro. In addition, decreased FOXM1 expression in SW620 cells reversed the acquisition of EMT phenotype by up-regulating E-cadherin, as well as reduction Vimentin and Snail expressions at protein and mRNA levels.

Conclusions: FOXM1 may regulate CRC cells metastasis through EMT program and FOXM1 may be a potential target for treatment of CRC.

Keywords: FOXM1, EMT, Metastasis, Colorectal cancer

\section{Background}

Colorectal cancer (CRC) is the third most common cancer and the third leading cause of cancer death in men and women in the United States. Although early detection tests and treatments have been improved in clinical practice, including modified surgical techniques and neoadjuvant chemotherapy combined with radiation therapy in CRC patients, the 5-year survival rate is decreasing to $12.5 \%$ in the advanced CRC patients who

\footnotetext{
* Correspondence: professorzxg@hotmail.com

${ }^{\dagger}$ Equal contributors

'Department of General Surgery, The First Affiliated Hospital of Soochow University, 215006 Suzhou, Jiangsu Province, China

Full list of author information is available at the end of the article
}

have metastasis of distant organs [1-3]. Therefore, there is an urgent need to identify novel prognostic hallmarks and to improve on current understanding of the molecular mechanisms of advanced CRC.

The transcription factor Forkhead box M1 (FOXM1) is an oncogenic transcription factor belongs to the FOX protein super family that shares an evolutionarily conserved winged helix DNA-blinding domain $[4,5]$. Largescale gene expression analysis by means of microarrays have demonstrated that FoxM1 is one of the most common overexpressed genes in a multitude of human solid tumors [6], including hepatocellular carcinomas [7], pancreatic cancer [8], breast cancer [9], ovarian cancer [10], colorectal cancer [11] and lung cancer [12], suggesting 
that FOXM1 is essential to regulate the tumorigenicity. Many studies have reported that FOXM1 is known as a key regulator of the cell cycle by regulating the transition from $G 1$ to $S$ and $G 2$ to $M$ phase and mitosis $[13,14]$, playing a positive effect on cell proliferation. Futhermore, enhanced expression of FoxM1 is associated with advanced stage, lymph node matastasis and acts as an independent prognostic factor in non-small cell lung cancer (NSCLC) [15]. Beyond that cell proliferation, FOXM1 also plays important roles in tumor angiogenesis, EMT, invasion, and metastasis [9,16-20]. The actual occurrence of EMT serves as a dominant role in invasion and metastasis of colon cancer [21], which is regulated by a various signal pathways, such as FOXM1PLAUR [22], FOXM1-caveolin-1 signaling pathway [23]. Emerging evidences suggest that enhanced FoxM1 levels lead to the acquisition of EMT phenotype, which contributes to tumor cell aggressiveness along with a series of molecule changes of epithelial or mesenchymal markers [24]. On the contrary, for example, downexpression of FOXM1 in RNAi-mediated gastric cancer cells reversed the EMT phenotype and upregulated the expression of epithelial markers E-cadherin, as well as downregulated the expression of mesenchymal markers ZEB1, ZEB2 and Vimentin [25]. However, the precise function and internal mechanisms of FOXM1 in colorectal cancer cells EMT and metastasis remain still indistinct.

In our present study, we detected the expression of FOXM1 in colorectal tumor tissue specimens by immunohistochemical staining from 87 CRC patients and investigated the relationships among mediated gene knockdown of FOXM1 on SW620 cells and EMT, proliferation, migration and invasion in vitro. Our results show that the downregulation of FoxM1 inhibits the cell migration, invasion, and proliferation of SW620 cells and reverses the EMT phenotype by up-regulating epithelial cell markers E-cadherin, as well as down-regulating the expression of the mesenchymal cell markers Vimentin and Snail at protein and mRNA levels. The results provide supportive evidence that FOXM1 may be an effective therapeutic target in CRC.

\section{Materials and methods}

Human colorectal cancer tissues and colon cancer cell lines Human colorectal cancer tissues were obtained from 87 patients at the Department of General Surgery, the First Affiliated Hospital of Soochow University from 20082013. Each tumor tissue and adjacent normal colon tissue (at least $2 \mathrm{~cm}$ distance from the tumor site) were collected from the same patient with a clear histological diagnosis of CRC who had received no any therapy before sample collection. The researches were supported by the Independent Ethics Committee (IEC) of the First
Affiliated Hospital of Soochow University and all patients were provided written informed consent. Human colon cancer cell lines HCT116, SW620, SW480, LOVO and DLD-1 were purchased from the Chinese Academy of Sciences (Shanghai, China). All five cell lines were maintained in DMED supplemented with 10\% fetal bovine serum (Sijiqing Biological Engineering Materials Co., Hangzhou, China) and cultured at $37^{\circ} \mathrm{C}$ in a humidified atmosphere containing $5 \% \mathrm{CO}_{2}$.

\section{Immunohistochemistry (IHC)}

The protein of FOXM1 of 87 human colorectal cancer tissue samples and adjacent normal colorectal tissues was detected through immunohistochemical staining (IHC). IHC was performed using the streptavidinperoxidase coujugate method. Surgical specimens were fixed in $10 \%$ formalin and embedded in paraffin. Briefly, the paraffin-embedded tissues were serially cut into $4 \mu \mathrm{m}$ sections, dewaxed, and rehydrated. Sections of paraffinembedded tissues were then blocked with peroxide in methanol, and nonspecific immunoglobulin binding was blocked by incubation with $10 \%$ normal goat serum for $15 \mathrm{~min}$. After rinsing with PBS, the sections were incubated at room temperature for $1 \mathrm{~h}$ with Foxm 1 polyclonal rabbit-anti-human antibody (Abcam, UK) at 1:200 dilution. After a PBS rinse, slides were then incubated for $25 \mathrm{~min}$ at room temperature with biotinylated goat-anti-rabbit immunoglobulin (1:1000, Zhongshan Biotechnology, China) followed by incubation with peroxidase-conjugated streptavidin for $20 \mathrm{~min}$. Finally, the slides were stained with fresh $0.05 \% 3,3^{\prime}$-diaminobenzidine (DAB), counterstained with hematoxylin, dehydrated, cleared in xylene, and fixed. Histological assessment was performed as described previously [26]. Immunostaining was independently examined by two clinical pathologists who were blinded of the patient outcome. Five high-power fields $(400 \times$ magnification) were randomly counted for each section. The brown staining on the cytoplasm was read as positive reactivity for FOXM1. The presence of brown colored granules on the cytoplasm was taken as a positive signal, and was divided by color intensity into not colored, light yellow, brown, tan and is recorded as 0,1 , 2,3 , respectively. We also chose five high-power fields from each slice and scored them. Positive cell rate of < $25 \%$ was a score of 1 , positive cell rate of $25 \sim 50 \%$ was a score of 2 , positive cell rate of $51 \sim 75 \%$ was a score of 3 , positive cell rate of $>75 \%$ was a score of 4 . The scores for FOXM1 positivity and staining intensity were multiplied to obtain a final score, which determines FOXM1 expression as $(-=0 ;+=1-4 ;++=5-8 ;+++=$ 9-12). In our current study, we classified all of the samples into the high expression group $(++$ or +++$)$ and the low expression group ( - or + ) according to the protein expression. 


\section{Short hairpin RNA transfection of human colon cancer cell line SW620}

Human FOXM1 shRNA (5'-GGACCACUUUCCCUA CUUU-3') and control-shRNA (5'-GGACCUGUAUGC GUACAUU-3') were synthesized by GenePharma (shanghai, china). SW620 cells were transfected with shFOXM1 or control-shRNA using Lipofectamine 2000 (Invitrogen, Life Technologies), according to the manufacturer's instructions.

\section{Quantitative real-time reverse transcription PCR (QRT-PCR)}

The mRNA expression of FOXM1, E-cadherin, Vimentin and Snail in SW620 cells after FOXM1-shRNA transfection were quantified by real-time RT-PCR. Total RNA was extracted from cells and tumor tissues using TRIzol Reagent (Invitrogen, Life Technologies) and cDNA was synthesized from $2 \mu \mathrm{g}$ of RNA using the First Strand cDNA Synthesis Kit (Fermentas) according to the manufacturer's instructions. QRT-PCR was carried out using Power SYBR ${ }^{\bullet}$ Green PCR Master Mix (ABI, USA) on the 7500 real time PCR system (ABI, life technology). The $\beta$-actin was used as a loading control for each specific gene. Each experiment was performed three times and each sample was tested in triplicate. The sequences for sense (S) and antisense (AS) primers as follows: human-foxm1-S, 5'-GGAGGAAATGCCACACTTAGCG3', human-foxm1-AS,5' -TAGGACTTCTTGGGTCTTGG GGTG-3', human-E-cadherin-S, 5'-CGGGAATGCAGTT GAGGATC-3', human-E-cadherin-AS,5'-AGGATGGTG TAAGCGATGGC-3', human-Vimentin-S,5' -GAGAACTT TGCCGTTGAAGC-3', human-Vimentin-AS,5' -GCTTCC TGTAGGTGGCAATC-3', human-Snail-S,5' -CTCTTTCC TCGTCAGGAAGC-3', human-Snail-AS,5'-GGCTGCTG GAAGGTAAACTC-3', $\beta$-actin-S,5'-CCACACTGTGCCC ATCTACG-3', $\beta$-actin-AS,5' -AGGATCTTCATGAGGT AGTCAGTCAG-3'. The PCR conditions consisted of $5 \mathrm{~min}$ at $95^{\circ} \mathrm{C} 1 \mathrm{cycle}, 30 \mathrm{sec}$ at $95^{\circ} \mathrm{C}, 30 \mathrm{sec}$ at $55^{\circ} \mathrm{C}$, $30 \mathrm{sec}$ at $72^{\circ} \mathrm{C}$ and $7 \mathrm{~min}$ at $72^{\circ} \mathrm{C} 40$ cycles. The $2^{-\Delta \Delta \mathrm{C}} \mathrm{T}$ method was applied to analyze the relative changes in gene expression [27].

\section{Protein extraction and western blot analysis}

Whole protein extracts from SW620 at $72 \mathrm{~h}$ following shRNA transfection or untransfection were lysed in icecold RIPA lysis buffer (Beyotime Inc., NanTong, China) according to manufacturer's protocol. From each sample preparation $50 \mu \mathrm{g}$ of whole protein was separated by SDS-PAGE and then transferred to PVDF membranes (Millipore, USA). Standard Western blotting was performed using a polyclonal rabbit antibody against human FoxM1 (1:1000, Abcam, UK), mouse anti-human Ecadherin (1:1000, Abcam, UK), rabbit anti-human Vimentin (1:1000, Abcam, UK), rabbit anti-human Snail (1:500, Abcam, UK) and rabbit anti- $\beta$-actin (1:1000., Beyotime, china). The signals from the primary antibody was amplified by HRP conjugated anti-mouse IgG or antirabbit IgG (1:1000; Beyotime, china) and detected with Enhanced Chemiluminescence Plus kit (Beyotime, China).

\section{MTT assays of cell viability}

Briefly, 24hours after transfection, SW620 cells of three groups were digested, re-suspended and seeded at a density of $4 \times 10^{3}$ cells/well in 96-well culture plates, After $24 \mathrm{~h}, 48 \mathrm{~h}$ and $72 \mathrm{~h}$ of incubation in complete medium, cells were added $20 \mu \mathrm{l}$ MTT solution $(5 \mathrm{mg} / \mathrm{ml})$ at $37^{\circ} \mathrm{C}$ for 4 hours. Then supernatants were removed and formazan crystals were dissolved in $150 \mu \mathrm{L}$ DMSO. After gentle shaking for 10 minutes, the absorbance (A) at $490 \mathrm{~nm}$ was measured by using a microplate reader. Each sample was four replicate wells and the experiment was repeated three times.

\section{Clonogenic assays}

Clonogenic assay was conducted to examine the effect of FoxM1-shRNA on cell growth in SW620 cells, as described previously [9]. $4 \times 10^{5}$ SW620 cells were plated in a 6 -well plate. After $24 \mathrm{~h}$ of transfection, the cells were trypsinized, and 1,000 single viable cells were plated in three 6-well plates. The cells were then incubated for 14 days at $37^{\circ} \mathrm{C}$ in the condition of $5 \% \mathrm{CO}_{2} / 5 \% \mathrm{O}_{2} / 90 \%$ $\mathrm{N}_{2}$. Colonies were stained with $0.1 \%$ crystal violet, washed with water, and counted ten random fields manually. The colonies containing at least 100 cells were scored. The surviving fraction in FoxM1-shRNA transfected SW620 cells was normalized to untreated control cells with respect to clonogenic efficiency.

\section{Wound healing assay}

Wound healing assay was adopted to test the migration ability of colon cancer cells. In our study, SW620 cells were digested after transfection by specific shRNA and control shRNA to human Foxm1 for $24 \mathrm{~h}$ in 6-well plates, $2 \times 10^{5}$ cells were plated in 24-well plates, when cell confluence reached approximately $100 \%$, the old medium was removed and the monolayer was wounded by scratching with a $10-\mu$ l sterile pipette tip lengthwise along the chamber, then cells were washed three times with PBS and cultured with serum-free medium at $37^{\circ} \mathrm{C}$. Images of cells migrating into the wound were photographed at $0 \mathrm{~h}, 24 \mathrm{~h}, 48 \mathrm{~h}$ and $72 \mathrm{~h}$ using an inverted microscope. Wound width $(\mu \mathrm{m})$ was measured using OpenLab software. Wound healing rate $=(0 \mathrm{~h}$ scratch width $-24 \mathrm{~h} / 48 \mathrm{~h}$ or $72 \mathrm{~h}$ scratch width)/0h scratch width $\times 100 \%$. The experiments were repeated three times. 


\section{Cell migration and invasion assays}

The migratory and invasive activity of the FoxM1 or control shRNA-transfected SW620 cells was tested using the Transwell chambers equipped with a pore size of $8 \mu \mathrm{m}$ (Corning, USA) according to the manufacturer's recommendations. After $24 \mathrm{~h}$ of transfection, $2 \times 10^{4}$ SW620 cells per well were resuspended in serum-free DMEM medium and seeded into the Transwell inserts either uncoated (for migration assay) or coated (for invasion assay) with growth factor-reduced Matrigel (BD Biosciences, Bedford, MA), whereas the lower chambers were filled with $500 \mu \mathrm{l}$ DMEM with $10 \%$ FBS. After $24 \mathrm{~h}$ incubation at $37^{\circ} \mathrm{C}$, the cells on the upper side of the insert filter were completely removed by wiping with a cotton swab, and the cells that had invaded were fixed in methanol and stained with $0.1 \%$ crystal violet. The cells were counted manually under an inverted microscope on five random fields (scale bar $=200 \mu \mathrm{m}$ ). Each experiment was repeated in triplicate.

\section{Statistical analysis}

Data are expressed as the means \pm standard deviation (SD). Significant differences between the groups were determined using the student's $t$-test and Chi-square test. A value of $\mathrm{P}<0.05$ was considered to indicate a statistically significant difference. All statistical analyses were performed with SPSS17.0 software (SPSS Inc, Chicago, IL, USA).

\section{Results}

FoxM1 is over-expressed in human colorectal cancer patients

To investigate whether FoxM1 is highly expressed in CRC tissues, we first detected the expression of FoxM1 protein in the 87 primary colorectal tumors and paired adjacent normal colorectal tissue specimens as well as invasive lymph nodes using immunohistochemical staining. Our immunostaining results showed a FoxM1positive staining in the nucleus and/or cytoplasm of colorectal tumor cells and invasive lymph nodes, whereas there was negative or weakly positive staining of FoxM1 in the paired adjacent normal colorectal tissues (Figure 1A-D). To further confirm the expression of FoxM1 in colon cancer, we detected the expression of FoxM1 in fresh colon cancer tissues, metastatic lymph nodes and adjacent normal tissues from four same patients. Real-time PCR was subjected to examine the mRNA levels. Consistent with the level of FoxM1 protein expression determined using immunostaining analyses, primary colorectal tumors and metastatic lymph nodes had significantly higher levels of FoxM1 mRNA expression than did surrounding normal colorectal tissue from 4 patients by QRT-PCR analysis, the difference between them was statistically significant (Figure $1 \mathrm{E} \mathrm{p}<0.01$ ). These results indicated that FoxM1 is commonly overexpressed in human colorectal tumors, particularly in metastatic lymph nodes.

\section{Relationship between Foxm 1 expression and clinicopathologic parameters in CRC patients}

To further assess the clinical relevance of FoxM1 overexpression in CRC patients, we then analyzed the relationship between the level of FoxM1 expression levels and clinicopathologic parameters in 87 CRC patients using immunohistochemical staining. As shown in Table 1, we observed that high expression of Foxm1 was significantly correlated with regional lymph nodes metastasis $\left(\chi^{2}=9.184, \mathrm{p}=0.002\right)$ and tumor recurrence $\left(\chi^{2}=8.025\right.$,

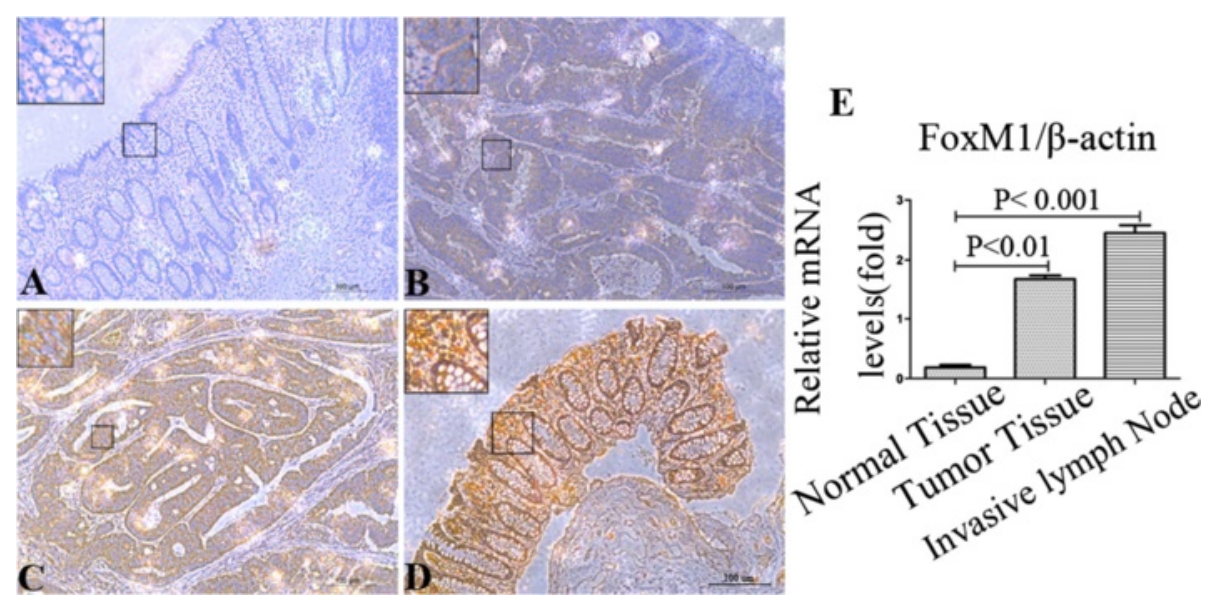

Figure 1 FoxM1 is highly expressed in human CRC patients. (A-D): Foxm1 protein expression in CRC cells, normal colon cells and invasive lymph nodes from immunohistochemical staining. The protein expression of FOXM1 was negative (A), mild positive (B), positive (C), strong positive (D), respectively. Scale bar $=100 \mu \mathrm{m}$. (E): QRT-PCR analyses of colon cancer tissues, invasive lymph nodes and adjacent normal tissues from 4 CRC patients. Foxm1 mRNA expression levels were significantly higher in colon cancer tissues and invasive lymph nodes than paired adjacent normal colon tissues. 
Table 1 Relationship between FoxM1 expression from IHC and clinicopathological factors of 87 CRC patients

\begin{tabular}{|c|c|c|c|c|c|}
\hline \multirow[t]{2}{*}{ Clinic parameters } & \multirow[t]{2}{*}{ Total } & \multicolumn{2}{|c|}{ FoxM1 expression } & \multirow[t]{2}{*}{$x^{2}$} & \multirow[t]{2}{*}{$P$ value } \\
\hline & & None or low & High & & \\
\hline Age(years) & $T=87$ & $\mathrm{~N}=35(40.2 \%)$ & $\mathrm{N}=52(59.8 \%)$ & & \\
\hline$<65$ & $33(37.9 \%)$ & $15(45.5 \%)$ & $18(54.5 \%)$ & 0.604 & 0.437 \\
\hline$>=65$ & $54(62.1 \%)$ & $20(37.0 \%)$ & $34(63.0 \%)$ & & \\
\hline \multicolumn{6}{|l|}{ Gender } \\
\hline Male & $42(48.3 \%)$ & 19(45.2\%) & $23(54.8 \%)$ & 0.847 & 0.357 \\
\hline Female & $45(51.7 \%)$ & 16(35.6\%) & $29(64.4 \%)$ & & \\
\hline \multicolumn{6}{|l|}{ Tumor size } \\
\hline$<5 \mathrm{~cm}$ & $56(64.4 \%)$ & $20(35.7 \%)$ & $36(64.3 \%)$ & 1.333 & 0.248 \\
\hline$>=5 \mathrm{~cm}$ & $31(35.6 \%)$ & $15(48.4 \%)$ & $16(51.6 \%)$ & & \\
\hline \multicolumn{6}{|l|}{ Tumor location } \\
\hline Right colon & 33(37.9\%) & $11(33.3 \%)$ & $22(66.7 \%)$ & 1.052 & 0.305 \\
\hline Left colon and rectum & $54(62.1 \%)$ & $24(44.4 \%)$ & $30(55.6 \%)$ & & \\
\hline \multicolumn{6}{|l|}{ TNM Stage } \\
\hline Stage $|/| \mid$ & $42(48.3 \%)$ & 19(45.2\%) & $23(54.8 \%)$ & 0.847 & 0.357 \\
\hline Stage III/IV & $45(51.7 \%)$ & 16(35.6\%) & $29(64.4 \%)$ & & \\
\hline \multicolumn{6}{|c|}{ Histological differentiation } \\
\hline Well & 28(32.2\%) & $12(42.9 \%)$ & 16(57.1\%) & 1.873 & 0.392 \\
\hline Moderate & $46(52.9 \%)$ & $20(43.5 \%)$ & $26(56.5 \%)$ & & \\
\hline Poor & 13(14.9\%) & $3(23.1 \%)$ & $10(76.9 \%)$ & & \\
\hline \multicolumn{6}{|c|}{ Lymph node metastasis } \\
\hline Yes & $47(54.0 \%)$ & $12(25.5 \%)$ & $35(74.5 \%)$ & 9.184 & $0.002^{* * *}$ \\
\hline No & $40(56.0 \%)$ & $23(57.5 \%)$ & $17(42.5 \%)$ & & \\
\hline \multicolumn{6}{|l|}{ Tumor recurrence } \\
\hline Yes & $53(60.9 \%)$ & $15(28.3 \%)$ & $38(71.7 \%)$ & 8.025 & $0.005^{* * *}$ \\
\hline No & 34(39.1\%) & $20(58.8 \%)$ & $14(41.2 \%)$ & & \\
\hline
\end{tabular}

$\mathrm{p}<0.001$ ); however, FoxM1 expression level was not associated with other clinicopathologic features, such as age, gender, tumor size, tumor location, TNM stages and histological differentiation (all $\mathrm{p}>0.05$ ).

\section{FoxM1 is up-regulated in CRC cell lines and associated} directly with migration ability of colorectal cancer cells To evaluate the baseline expression levels of FoxM1 in a series of human colorectal cancer cell lines, including HCT116, LOVO, DLD-1, SW480, SW620, we detected the mRNA and protein expression of FoxM1 by realtime PCR and Western Blot analyses, respectively. Our studies evidenced that SW620 cells showed the highest expression of FoxM1, especially in relation to the SW480 cell line (Figure 2A\&B). To further test whether the expression levels of FoxM1 were related to migration ability of CRC cells, we measured the effects of FoxM1 expression levels on cancer cell migration by transwell systems between SW480 and SW620 cells; we found that the numbers of migratory SW620 cells were more dramatically higher than those of SW480 cells (Figure 2C, $\mathrm{P}<0.001)$.

Deletion of FoxM1 gene down-regulated the mRNA and protein levels of FoxM1 expression in SW620 cells

In order to explore the effect the shRNA silencing FoxM1 expression in SW620 cells, SW620 cells were transfected with the FoxM1 shRNA (shFoxM1) and control-shRNA; after $72 \mathrm{~h}$, the total protein and RNA of all transfected and untransfected cells were extracted and analyzed by Western blots and real-time PCR. As shown in Figure 3A-C, FoxM1 expression was distinctly decreased at mRNA and protein levels in transfected cells with FoxM1 shRNA compared with control-shRNA transfected cells and untreated cells. The results showed that the specific shRNA to FoxM1 effectively suppressed the expression of FoxM1 in SW620 cells. 


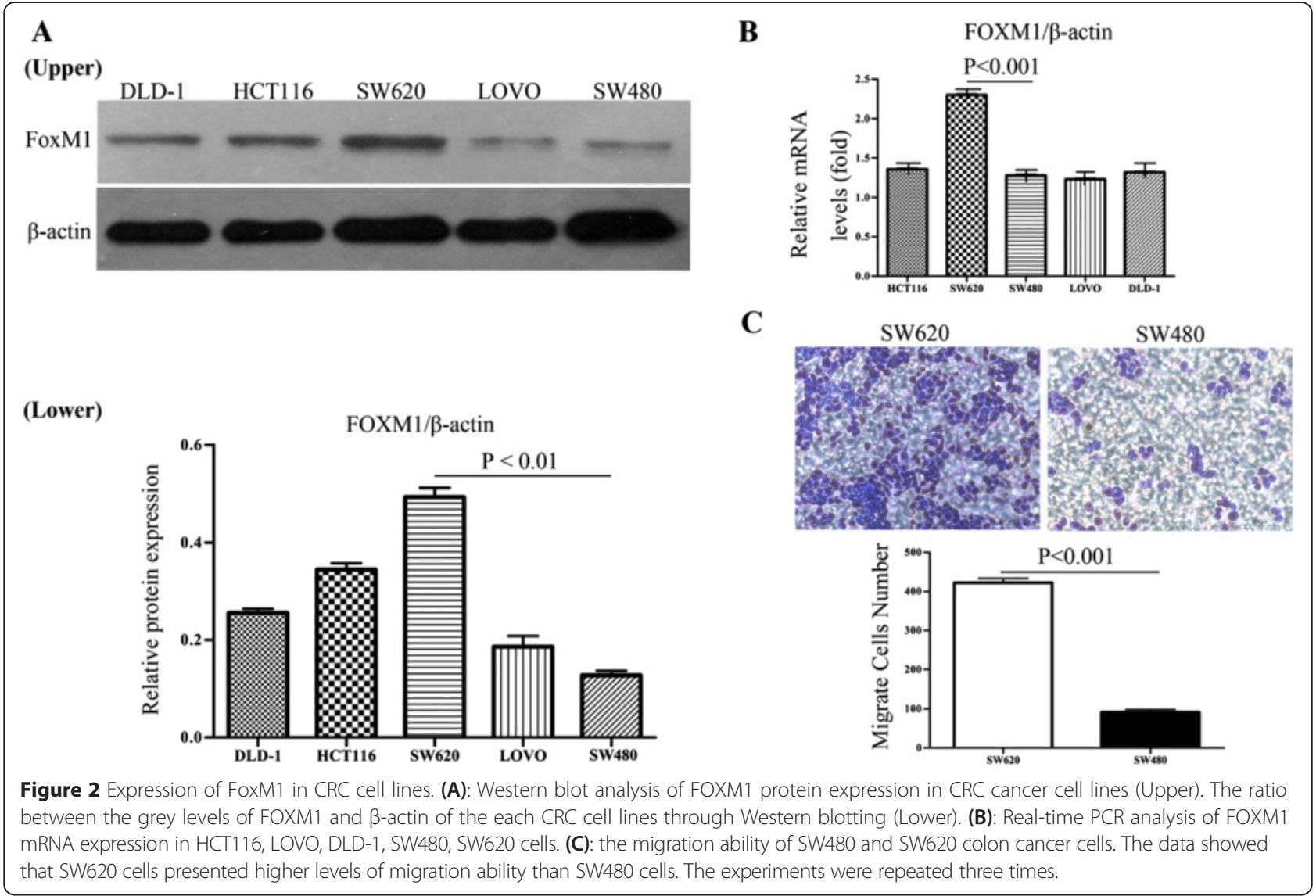

\section{Down-expression of FoxM1 inhibited the proliferation and clonogenicity in SW620 cells}

In order to determine the effect of FoxM1 on the proliferation of SW620 cells in vitro, the proliferation curves were detected by MTT assays at $24 \mathrm{~h}, 48 \mathrm{~h}, 72 \mathrm{~h}$ after $24 \mathrm{~h}$ of transfection. We found that SW620 cells which down-regulated the FoxM1 expression experienced a significant inhibition of cell viability compared with control-shRNA and untreated cells respectively (Figure 4A, both $\mathrm{P}<0.01$ ).

To further examine whether the knockdown of FoxM1 gene reduces the clonogenic formation of SW620 cells, after $24 \mathrm{~h}$ of tranfection, $2 \times 10^{3} /$ well of SW620 cells were plated in 6-well plates and cultured 14days to observe the numbers of colony formation. The results showed that the numbers of colonies in FoxM1depleted SW620 cells displayed an apparent reduction compared with control-shRNA and untreated cells respectively (Figure 4B, $\mathrm{p}<0.05$ ).

\section{Knockdown of FoxM1 expression by shRNA induced a cellular morphologic change and EMT-related mRNA and protein expressions in SW620 cells}

As illustrated in Figure 5A, SW620 cancer cells have a typical mesenchymal morphology characterized by elongated, spindle-shaped phenotype. To examine whether downregulation of FoxM1 expression could change cell morphology, we transfected FoxM1 shRNA (shFOXM1) and control shRNA into SW620 cells; after 72hours of transfection, we found that in the monolayer culture system, SW620 cellular morphology changed from an elongated, spindle-shaped, mesenchymal phenotype to a more rounded, epithelial-like phenotype after knockdown of FoxM1 expression and observation under the microscopy. The results indicated that knockdown of FoxM1 expression altered mesenchymal morphology of SW620 cells and reversed the typical epithelial-tomesenchymal transition.

To further understand the internal molecular mechanism of mesenchymal-to-epithelial transition when FoxM1 was down-expressed, we investigated the EMT-related protein expression levels in transfected and untransected SW620 cells by western blotting (Figure 5B). The knockdown of FoxM1 gene by shRNA led to markedly reduce the mesenchymal marker Vimentin and Snail protein expression, whereas significantly increased the protein level of E-cadherin, which is a typical epithelial marker. Consistent with the real-time PCR analysis (Figure 5C-E), the down-regulation of FoxM1 expression significantly enhanced E-cadherin expression and attenuated Vimentin 


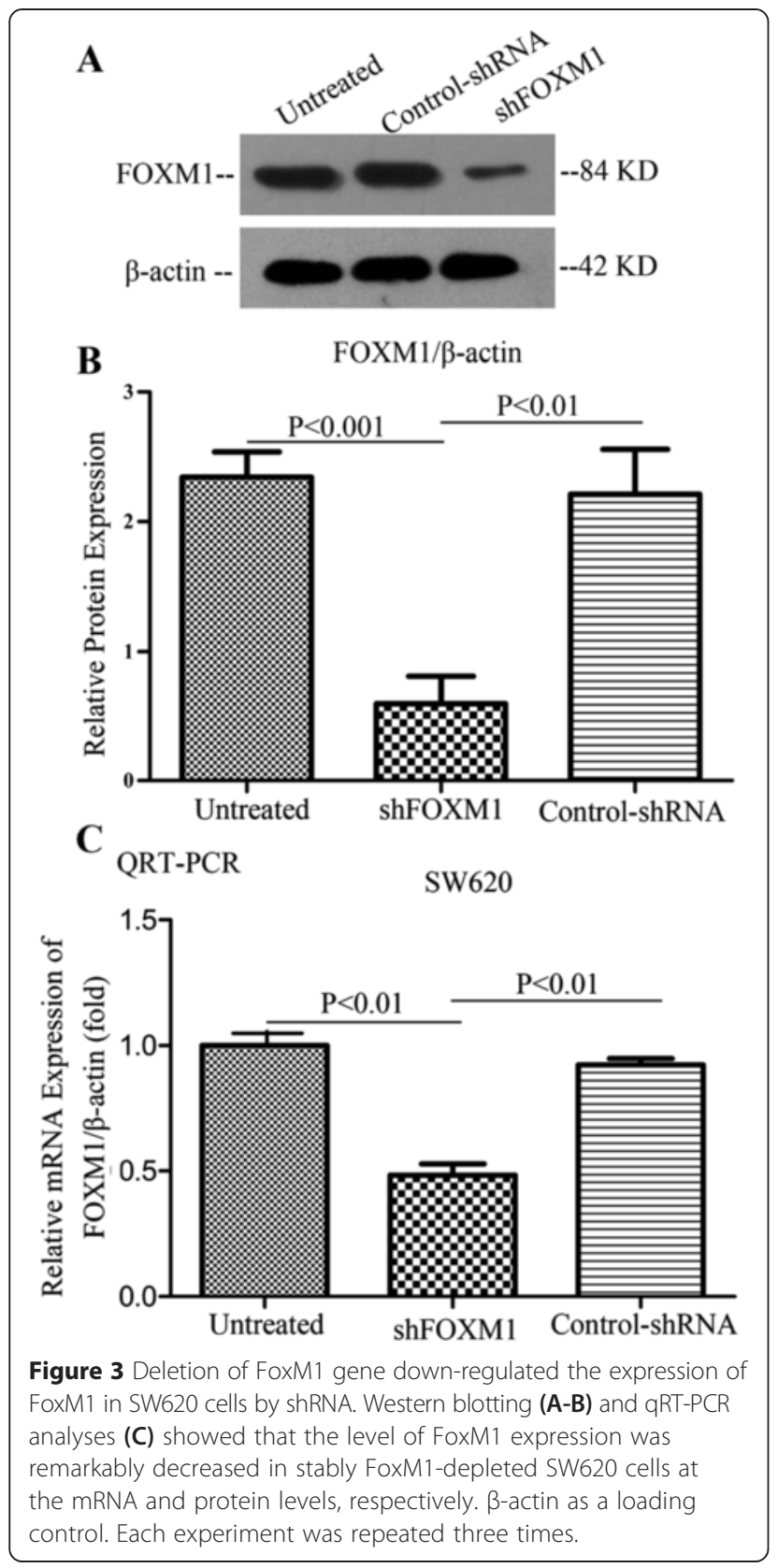

and Snail expression in FoxM1-knockdown SW620 cancer cells compared with those of control cells at the mRNA levels. The results demonstrated that FoxM1 played an important role in epithelial-to-mesenchymal transition regulation in CRC.

\section{Effect of altered FoxM1 expression on SW620 colon cancer cells migration and invasion in vitro}

Previous studies had suggested that down-expression of FoxM1 promoted E-cadherin expression and suppressed Vimentin and Snail expression, which were pronouncedly associated with EMT regulation. To determine whether attenuated FoxM1 expression reversed the
EMT process and further inhibited the migration and invasion in the colorectal cancer cells, we first explored the role of FoxM1 in CRC cell migration via the wound healing assay. The FoxM1-decreasing SW620 cells showed comparatively slower migration towards the wound space; however, control shRNA cells and untreated cells migrated aggressively and nearly closed the wound at $72 \mathrm{~h}$ after transfection (Figure $6 \mathrm{~A}-\mathrm{B},{ }^{* * *} \mathrm{p}<0.01$ ). Similar results arosed in the transwell migration system (Figure 6C-left, D-upper, ${ }^{* * *} \mathrm{P}<0.001$ ).

Consistent with the impact of altered FOXM1 expression on the invasion of SW620 cancer cells in vitro, transwell invasion assay revealed that FoxM1 shRNAtransfected cells showed a low level of penetration through the Matrigel-coated membrane compared with the control cells (Figure $6 \mathrm{C}$-right, $\mathrm{D}$-lower, ${ }^{* * * *} \mathrm{P}<0.001$ ). All these results clearly show that FoxM1 participates in EMT process and promotes the migration and matastasis in colorectal cancer cells.

\section{Discussion}

FOXM1 is commonly regarded as an oncogenic transcription factor and abnormal expression and activation of FOXM1 is associated with the proliferation and metastasis of human colon cancer cells, as an independent poor prognostic factor and conversely correlated with poor OS and MFS in CRC patients $[11,17,28]$. In the present study, FOXM1 protein expression was examined using immunohistochemisty analysis in CRC patients and we detected that FOXM1 was highly expressed in most human primary CRC tissues, particularly in invaded lymph nodes, whereas lowly expressed in adjacent normal colon tissues. Meanwhile, the strong relationships among FOXM1 expression and clinicopathology features had been investigated in CRC patients by IHC, our results indicated that FOXM1 overexpression was significantly associated with regional lymph nodes metastasis and tumor recurrence, suggesting important roles of FOXM1 in human colorectal cancer tumorigenesis and distant metastasis. The results show that FOXM1 has the potential to be a novel therapeutic target in CRC.

A mounting body of evidence notes that FOXM1 is a critical regulator of both the G1/S and G2/M transitions through the cell cycle progression, which is required for proliferative expansion during tumor progression [5]. Knockdown of FoxM1 inhibits expression of JNK1 and cyclinA2, which are involved in G1-S progression and in accumulation of cells in G2/M [29]. Previous research had revealed the role of FOXM1 in cell growth; increased expression of Foxm1 in Rosa26-Foxm1b transgenic mice directly regulated the cell-cycle progression of colon tumor cells by promoting S-phase progression and entry into mitosis [30]. Consistently, our current studies suggested that inhibition of FOXM1 expression 


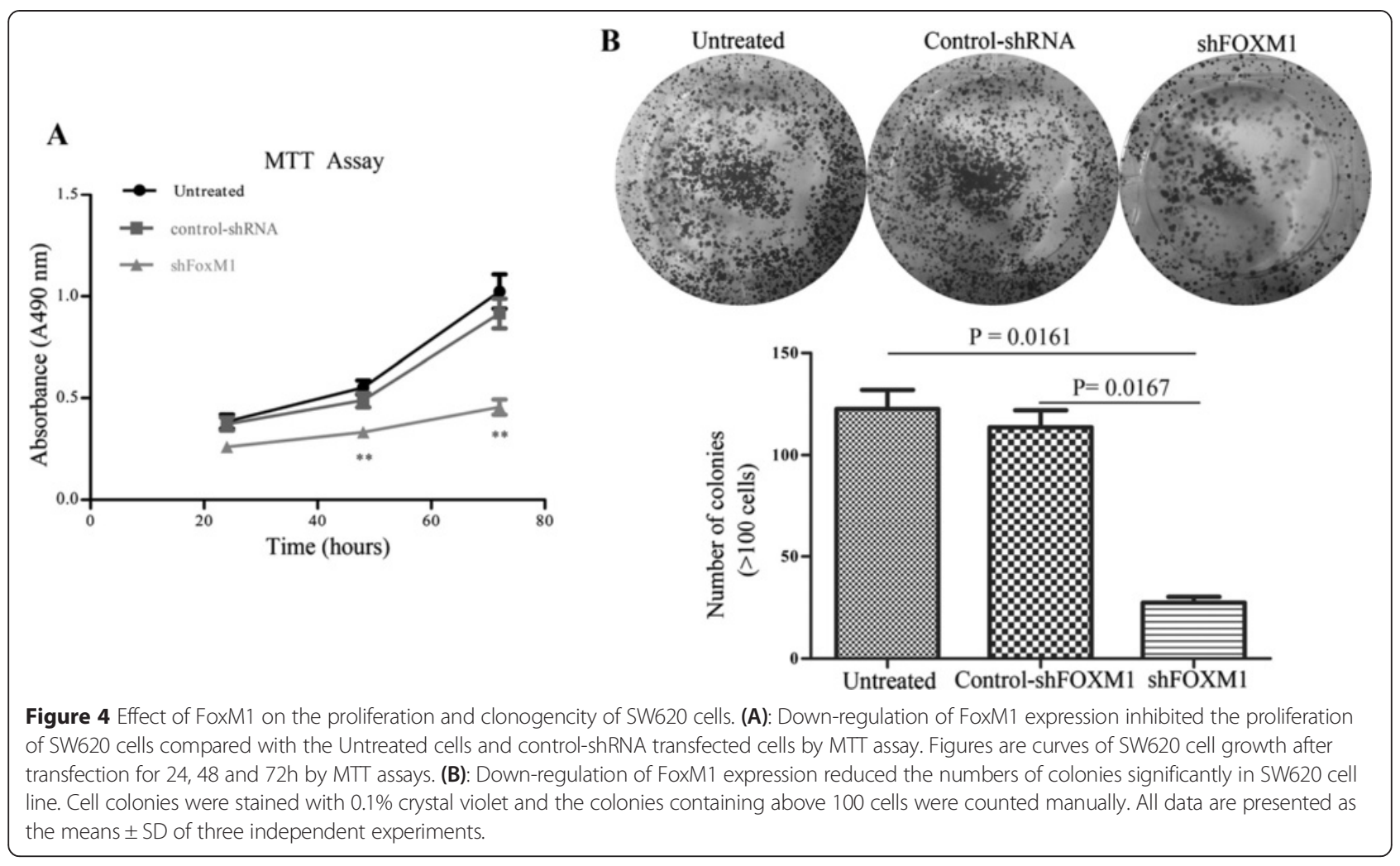

in SW620 cells by shRNA transfection decreased the proliferation and colony forming capability compared with that control-shRNA SW620 cells. Besides the cell proliferation capability of FOXM1, aberrant regulation of FOXM1 is a leading factor of malignancy tumor metastasis [10]. In our five CRC cell lines, HCT116, SW620, SW480, LOVO, DLD-1, western blot and qRTPCR analyses showed that FOXM1 expression levels were significantly increased, especially in SW620 cells. Meanwhile, we found that SW620 cells exhibited the much higher metastatic ability, revealing that FOXM1 overexpression may promote the tumor metastasis.

As shown in our wound healing assays and transwell migration/invasion systems, down-expression of FOXM1 remarkly inhibited the wound healing, migration and invasion of FOXM1-depleted SW620 cells. All our in vitro assays suggest a pivotal role for the FOXM1 in the metastasis progression of colorectal cancer and FOXM1 silencing could efficiently control the CRC cells metastasis.

Metastasis of tumor is a multistep and complex process including local invasion, intravasation, survival in circulation, extravasation, micrometastasis formation and metastatic colonization [31]. For tumor cells metastatic programs, the expression of FOXM1 may regulate a series of interrelated events including the expession of caveolin1 [23], VEGF [17,18], MPP-2 and MPP-9 [19], which are important to epithelial-mesenchymal transition (EMT), angiogenesis and metastasts. Importantly, a mounting body of work suggests that EMT, by which epithelial cells acquire mesenchymal characteristics leading to increased migratory and invasive potential of the cancer cells, has a critical role in metastasis [32,33]. Recently, numerous observations support the idea that the occurrence of EMT is closely associated with FOXM1 signaling activation. EMT has been reported as a pivotal program in several human solid cancers, including CRC [34], gastric cancer [25] and breast cancer [16], which is considered as the first step of tumor invasion and metastasis. Recent research shows that FOXM1c overexpression upregulates UPAR expression in pancreatic cancer cells and promotes EMT. Contrariwise, inhibition of FOXM1c expression suppressed UPAR expression by siRNA; furthermore, FOXM1c silencing expression could be targeted to reverse the acquiring of EMT phenotype in L3.7 cells [35]. Our present findings are consistent with these previous results. we transfected specific shRNA to FOXM1 into SW620 cells, which have a typical mesenchymal phenotype and high expression of FOXM1. We found that this transfection greatly inhibited FOXM1 mRNA and protein expression. Additionally, the SW620 cells reversed the acquisition of the EMT phenotype, changing from an elongated, spindle-shaped, mesenchymal phenotype to a more rounded, epithelial-like phenotype. As shown in recent studies, targeting FoxM1 signaling by novel small interference RNA silencing technique or miR-200 family members would be useful for reversing the EMT phenotype, which would likely result in 


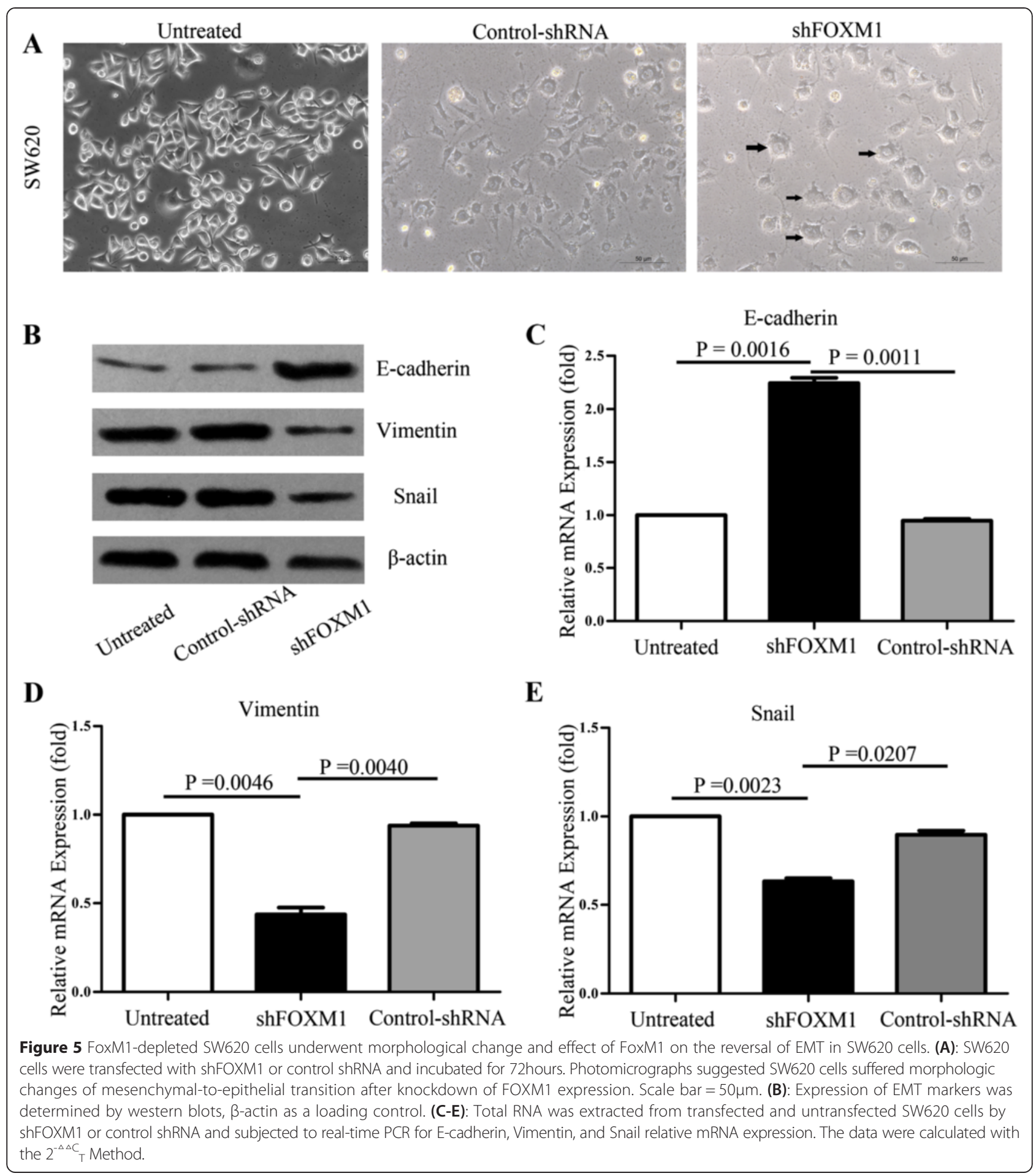

the reversal of drug resistance and elimination of cancer cells [24]. Interestingly, knockdown expression of FOXM1 down-regulated mesenchymal cell markers Vimentin and Snail and up-regulated the epithelial cell marker Ecadherin, which acted as key regulators in the process of epithelial-to-mesenchymal transition (EMT). Emerging evidences suggest that many EMT-inducing transcription factors such as Snail and ZEB1 have been found to be associated with expression of FOXM1 [15,24,36], which leads to tumor aggressiveness and metastasis. E-cadherin is a typical epithelial marker and loss of E-cadherin is a significant hallmark of EMT and can be mediated by the repressor binding directly to E-box motifs within the proximal E-cadherin promoter, such as snail $[37,38]$. 


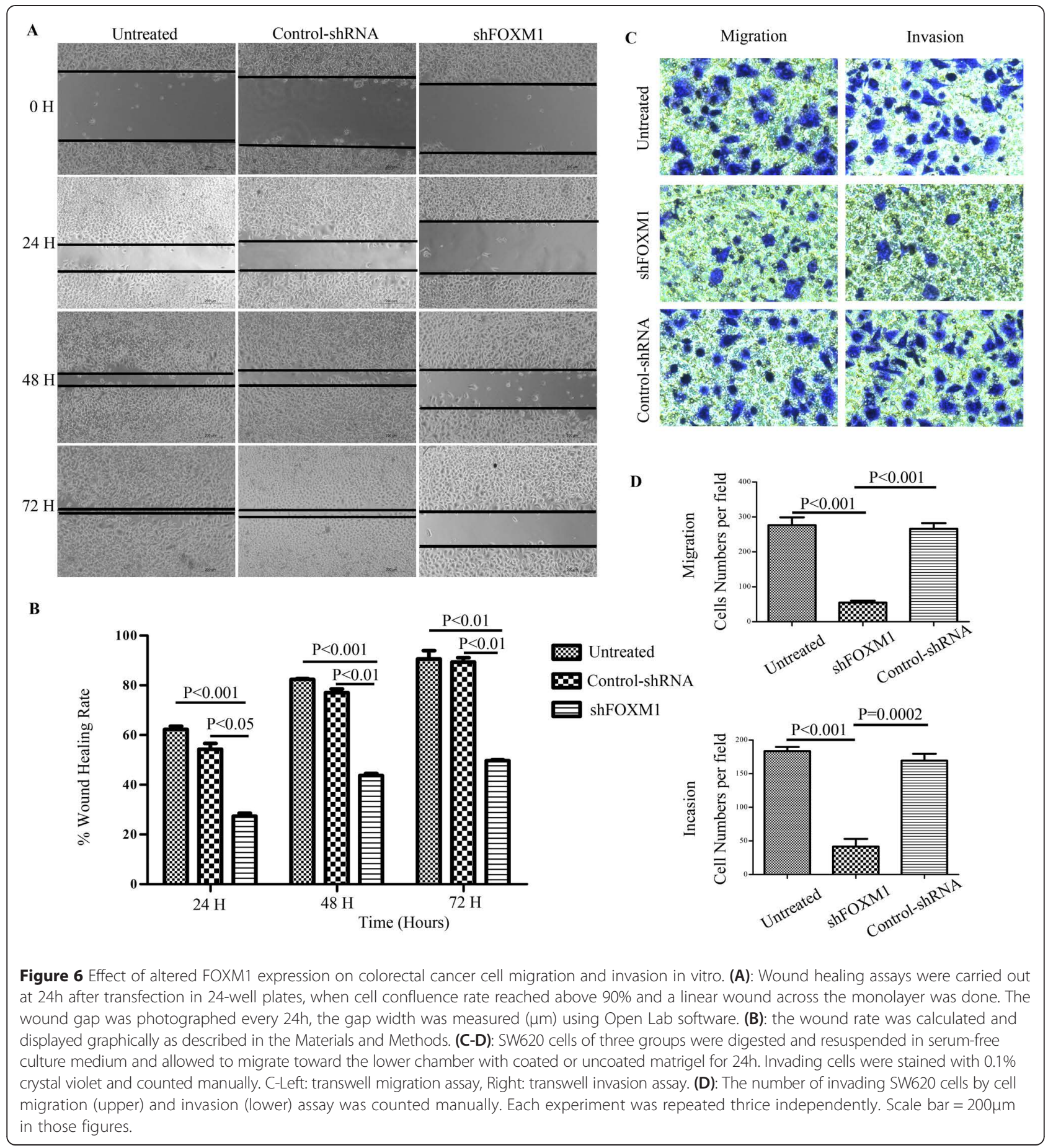

Snail is one of zinc-finger transcription factors that have been known as an essential player in the aggressive phenotype of EMT $[39,40]$. Increasing evidence suggests that Snail acts as a critical role during embryonic development and is essential for the formation of the fibroblasts during inflammation [41-43]. Moreover, Snail is highly expressed in CRC, especially in lymph node metastasis of CRC [44]. Consistent with our studies,
FOXM1 has an intimate relationship with the expression of Snail in CRC cells. Recent studies demonstrated that ectopic FOXM1 activation increased Snail activity through AKT signaling in hepatocellular carcinoma cells isolated from FoxM1b Tg;Arf-/- mice. Increased FoxM1b levels induce EMT by activating AKT and subsequently increasing GSK-3 $\beta$, which enhanced Snail expression [45]. In addition, new researches have verified that Foxm1 directly 
bound to and increased activity of Snail1 promoter, namely Snail1 is a direct downstream transcriptional target of Foxm 1 in the radiation-induced pulmonary fibrosis and lung adenocarcinoma progression $[43,46]$. Taken together, our current study strongly suggests that FOXM1 signaling has important roles in CRC cells proliferation and aggressiveness; thus, it is indicated that an intervening strategy targeting FOXM1 signaling in colon cancer may be of clinical value.

\section{Conclusions}

In summary, our studies have demonstrated that overexpression of FOXM1 is associated with distant lymph metastasis and tumor recurrence, and knockdown of FOXM1 by shRNA inhibited cell proliferation, migration and invasion of human colon cancer cells. In addition, we determined that downexpression of FOXM1 reversed the acquisition of EMT phenotype and upregulated the expression of E-cadherin, while decreasing Snail and Vimentin expression. Based on these studies, we conclude that FOXM1 may serve as a promising therapeutic target of CRC.

\section{Competing interests}

The authors declare that they have no competing interests.

\section{Authors' contributions}

KKY carried out literature research, experimental studies and data acquisition, participated in the study design and drafted the manuscript. LHJ performed the clinic cases collection and carried out the data acquisition, participated in the study design and helped to draft the manuscript. YH and JY participated in the design of the study and performed the statistical analyses. HFC and YZY helped to western blots, real-time PCR and data acquisition. XGZ proposed the study and participated in its design and helped to draft, and assisted writing the manuscript. All authors had already read and approved the final manuscript.

\section{Acknowledgements}

This study was supported by the Project of Medical Science and Technology Development Foundation of Jiangsu Province (Grant No. H201209), the Project of Nature Science Foundation of China (Grant No. 81201905), the Nature Science Research Grants of the University of Jiangsu Province (Grant No. 12KJB320009), the Nature Science Research Grants of the University of Jiangsu Province (Grant No. 13KJB320019) and the Science and Technology Research Project in the Science and Technology Bureau of Suzhou City (Grant No. SYS201220)

\section{Author details}

1Department of General Surgery, The First Affiliated Hospital of Soochow University, 215006 Suzhou, Jiangsu Province, China. ${ }^{2}$ Department of Laparoscopic Surgery, The First Affiliated Hospital of Soochow University, 215006 Suzhou, Jiangsu Province, China.

Received: 27 January 2015 Accepted: 14 April 2015

Published online: 03 May 2015

\section{References}

1. Siegel R, DeSantis C, Jemal A. Colorectal cancer statistics, 2014. CA Cancer J Clin. 2014;64:104-17.

2. DeSantis CE, Lin CC, Mariotto AB, Siegel RL, Stein KD, Kramer JL, et al. Cancer treatment and survivorship statistics, 2014. CA Cancer J Clin. 2014:64:252-71.

3. Ferlay J, Shin HR, Bray F, Forman D, Mathers C, Parkin DM. Estimates of worldwide burden of cancer in 2008: GLOBOCAN 2008. Int J Cancer. 2010;127:2893-917.
4. Halasi M, Gartel AL. FOX (M1) news-It is cancer. Mol Cancer Ther. 2013;12:245-54

5. Kalin TV, Ustiyan V, Kalinichenko W. Multiple faces of FoxM1 transcription factor. Cell Cycle. 2011;10:396-405.

6. Pilarsky C, Wenzig M, Specht T, Saeger HD, Grützmann R. Identification and validation of commonly overexpressed genes in solid tumors by comparison of microarray data. Neoplasia. 2004;6:744-50.

7. Kalinichenko W, Major ML, Wang X, Petrovic V, Kuechle J, Yoder HM, et al. Foxm $1 b$ transcription factor is essential for development of hepatocellular carcinomas and is negatively regulated by the p19ARF tumor suppressor. Genes Dev. 2004;18:830-50.

8. Wang Z, Ahmad A, Banerjee S, Azmi A, Kong D, Li Y, et al. FoxM1 is a novel target of a natural agent in pancreatic cancer. Pharm Res. 2010;27:1159-68.

9. Ahmad A, Wang Z, Kong D, Ali S, Li Y, Banerjee S, et al. FoxM1 downregulation leads to inhibition of proliferation, migration and invasion of breast cancer cells through the modulation of extra-cellular matrix degrading factors. Breast Cancer Res Treat. 2010;122:337-46.

10. Wen N, Wang $Y$, Wen L, Zhao S-H, Ai Z-H, Wang Y, et al. Overexpression of FOXM1 predicts poor prognosis and promotes cancer cell proliferation, migration and invasion in epithelial ovarian cancer. J Transl Med. 2014;12:134.

11. Uddin S, Ahmed M, Hussain A, Abubaker J, Al-Sanea N, AbdulJabbar A, et al. Genome-wide expression analysis of Middle Eastern colorectal cancer reveals FOXM1 as a novel target for cancer therapy. Am J Pathol. 2011;178:537-47.

12. Kim I-M, Ackerson T, Ramakrishna S, Tretiakova M, Wang I-C, Kalin TV, et al. The Forkhead Box $\mathrm{m} 1$ transcription factor stimulates the proliferation of tumor cells during development of lung cancer. Cancer Res. 2006;66:2153-61.

13. Wierstra I, Alves J. FOXM1, a typical proliferation-associated transcription factor. Biol Chem. 2007;388:1257-74

14. Leung TW, Lin SS, Tsang AC, Tong CS, Ching JC, Leung WY, et al. Overexpression of FoxM1 stimulates cyclin B1 expression. FEBS Lett. 2001;507:59-66.

15. Kong F-F, Qu Z-Q, Yuan H-H, Wang J-Y, Zhao M, Guo Y-H, et al. Overexpression of FOXM1 is associated with EMT and is a predictor of poor prognosis in non-small cell lung cancer. Oncol Rep. 2014;31:2660-8.

16. Yang C, Chen H, Tan G, Gao W, Cheng L, Jiang X, et al. FOXM1 promotes the epithelial to mesenchymal transition by stimulating the transcription of Slug in human breast cancer. Cancer Lett. 2013;340:104-12.

17. Li Q, Zhang N, Jia Z, Le X, Dai B, Wei D, et al. Critical role and regulation of transcription factor FoxM1 in human gastric cancer angiogenesis and progression. Cancer Res. 2009;69:3501-9.

18. Zhang Y, Zhang N, Dai B, Liu M, Sawaya R, Xie K, et al. FoxM1B transcriptionally regulates vascular endothelial growth factor expression and promotes the angiogenesis and growth of glioma cells. Cancer Res. 2008;68:8733-42

19. Wang Z, Banerjee S, Kong D, Li Y, Sarkar FH. Down-regulation of Forkhead Box M1 transcription factor leads to the inhibition of invasion and angiogenesis of pancreatic cancer cells. Cancer Res. 2007;67:8293-300.

20. Chen H, Zou Y, Yang H, Wang J, Pan H. Downregulation of FoxM1 inhibits proliferation, invasion and angiogenesis of HeLa cells in vitro and in vivo. Int J Oncol. 2014:45:2355-64.

21. Bates RC, Mercurio AM. Review the epithelial-mesenchymal transition (EMT) and colorectal cancer progression. Cancer Biol Ther. 2005;4:365-70.

22. Li D, Wei P, Peng Z, Huang C, Tang H, Jia Z, et al. The critical role of dysregulated FOXM1-PLAUR signaling in human colon cancer progression and metastasis. Clin Cancer Res. 2013;19:62-72.

23. Huang C, Qiu Z, Wang L, Peng Z, Jia Z, Logsdon CD, et al. A novel FoxM1caveolin signaling pathway promotes pancreatic cancer invasion and metastasis. Cancer Res. 2012;72:655-65.

24. Bao B, Wang Z, Ali S, Kong D, Banerjee S, Ahmad A, et al. Over-expression of FoxM1 leads to epithelial-mesenchymal transition and cancer stem cell phenotype in pancreatic cancer cells. J Cell Biochem. 2011;112:2296-306.

25. Miao L, Xiong X, Lin Y, Cheng Y, Lu J, Zhang J, et al. Down-regulation of FoxM1 leads to the inhibition of the epithelial-mesenchymal transition in gastric cancer cells. Cancer Gene. 2014;207:75-82.

26. He S-B, Yuan Y, Wang L, Yu M-J, Zhu Y-B, Zhu X-G. Effects of cyclindependent kinase 8 specific siRNA on the proliferation and apoptosis of colon cancer cells. J Exp Clin Cancer Res. 2011;30:109.

27. Livak KJ, Schmittgen TD. Analysis of relative gene expression data using real-time quantitative PCR and the 2(-Delta Delta C(T)) Method. Methods. 2001;25:402-8. 
28. Chu X-Y, Zhu Z-M, Chen L-B, Wang J-H, Su Q-S, Yang J-R, et al. FOXM1 expression correlates with tumor invasion and a poor prognosis of colorectal cancer. Acta Histochem. 2012;114:755-62.

29. Wang I-C, Chen Y-J, Hughes DE, Ackerson T, Major ML, Kalinichenko W, et al. FoxM1 regulates transcription of JNK1 to promote the G1/S transition and tumor cell invasiveness. J Biol Chem. 2008;283:20770-8.

30. Yoshida Y, Wang I, Yoder HM, Davidson NO, Costa RH. The forkhead box M1 transcription factor contributes to the development and growth of mouse colorectal cancer. Gastroenterology. 2007;132:1420-31.

31. Valastyan S, Weinberg RA. Tumor metastasis: molecular insights and evolving paradigms. Cell. 2011;147:275-92.

32. Thiery JP, Sleeman JP. Complex networks orchestrate epithelialmesenchymal transitions. Nat Rev Mol Cell Biol. 2006;7:131-42.

33. Yue D, Li H, Che J, Zhang Y, Tseng H-HK, Jin JQ, et al. Hedgehog/Gli promotes epithelial-mesenchymal transition in lung squamous cell carcinomas. J Exp Clin Cancer Res. 2014;33:1-7.

34. Loboda A, Nebozhyn MV, Watters JW, Buser CA, Shaw PM, Huang PS, et al. EMT is the dominant program in human colon cancer. BMC Med Genomics. 2011;4:9.

35. Huang C, Xie D, Cui J, Li Q, Gao Y, Xie K. FOXM1c promotes pancreatic cancer epithelial-to-mesenchymal transition and metastasis via upregulation of expression of the urokinase plasminogen activator system. Clin Cancer Res. 2014;20:1477-88.

36. Yang J, Weinberg RA. Epithelial-mesenchymal transition: at the crossroads of development and tumor metastasis. Dev Cell. 2008;14:818-29.

37. Batlle E, Sancho E, Francí C, Domínguez D, Monfar M, Baulida J, et al. The transcription factor snail is a repressor of E-cadherin gene expression in epithelial tumour cells. Nat Cell Biol. 2000;2:84-9.

38. Dong C, Wu Y, Yao J, Wang Y, Yu Y, Rychahou PG, et al. G9a interacts with snail and is critical for snail-mediated E-cadherin repression in human breast cancer. J Clin Invest. 2012;122:1469-86.

39. Wu Y, Zhou BP. Snail: more than EMT. Cell Adh Migr. 2010;4:199-203.

40. Kaufhold S, Bonavida B. Central role of snail1 in the regulation of EMT and resistance in cancer: a target for therapeutic intervention. J Exp Clin Cancer Res. 2014;33:62.

41. Wang H, Wang H-S, Zhou B-H, Li C-L, Zhang F, Wang X-F, et al. Epithelialmesenchymal transition (EMT) induced by TNF-a requires AKT/GSK-3ßmediated stabilization of snail in colorectal cancer. PLoS One. 2013:8:e56664.

42. Barrallo-Gimeno A, Nieto MA. The snail genes as inducers of cell movement and survival: implications in development and cancer. Development. 2005;132:3151-61.

43. Balli D, Ustiyan V, Zhang Y, Wang I, Masino AJ, Ren X, et al. Foxm1 transcription factor is required for lung fibrosis and epithelial-tomesenchymal transition. EMBO J. 2013;32:231-44.

44. Fan X-J, Wan X-B, Yang Z-L, Fu X-H, Huang Y, Chen D-K, et al. Snail promotes lymph node metastasis and twist enhances tumor deposit formation through epithelial-mesenchymal transition in colorectal cancer. Hum Pathol. 2013:44:173-80.

45. Park HJ, Gusarova G, Wang Z, Carr JR, Li J, Kim KH, et al. Deregulation of FoxM1b leads to tumour metastasis. EMBO Mol Med. 2011;3:21-34.

46. Wei $P$, Zhang $N$, Wang $Y$, Li D, Wang $L$, Sun $X$, et al. FOXM1 promotes lung adenocarcinoma invasion and metastasis by upregulating SNAlL. Int J Biol Sci. 2015;11:186-98.

\section{Submit your next manuscript to BioMed Central and take full advantage of:}

- Convenient online submission

- Thorough peer review

- No space constraints or color figure charges

- Immediate publication on acceptance

- Inclusion in PubMed, CAS, Scopus and Google Scholar

- Research which is freely available for redistribution 